Preprint typeset in JHEP style - HYPER VERSION

UB-ECM-PF-09/01

\title{
On Schwinger Pair Creation in Gravity and in Closed Superstring Theory
}

\author{
Jorge G. Russo \\ Institució Catalana de Recerca i Estudis Avançats (ICREA), \\ Institute of Cosmos Sciences and Department ECM, University of Barcelona, \\ Av.Diagonal 647, Barcelona 08028 SPAIN
}

\begin{abstract}
We investigate the Schwinger pair creation process in the context of gravitational models with the back reaction of the electric field included in the geometry. The background is also an exact solution of type II superstring theory, where the electric field arises by Kaluza-Klein reduction. We obtain a closed formula for the pair creation rate that incorporates the gravitational back reaction. At weak fields it has the same structure as the general Schwinger formula, albeit pairs are produced by a combination of Schwinger and Unruh effect, the latter due to the presence of a Rindler horizon. In four spacetime dimensions, the rate becomes constant at strong electric fields. For states with mass of Kaluza-Klein origin, the rate has a power-like dependence in the electric field, rather than the familiar (non-perturbative) exponential dependence. We also reproduce the same formula from the string partition function for winding string states. Finally, we comment on the generalization to excited string states.
\end{abstract}

KEYwORDS: Schwinger effect, Gravity, String theory. 


\section{Contents}

1. Introduction

2. Charged scalar field 1

2.1 Electric field in Minkowski spacetime 1

2.2 Electric field in Rindler space

3. Electric field in a gravitational theory

3.1 Calculation of the pair-production rate 8

3.2 General properties 11

3.3 Discussion 13

4. Bosonic string model 14

4.1 Schwinger effect for winding string states 15

4.2 Comments on the extension to general string states 17

\section{Introduction}

The Schwinger effect of pair creation in the presence of an electric field is represented by a probability given by the formula [1]

$$
\mathcal{W}=T V_{d-1} \frac{2 j+1}{(2 \pi)^{d-1}} \sum_{k=1}^{\infty}(-1)^{(2 j+1)(k+1)}\left(\frac{e E}{k}\right)^{\frac{d}{2}} e^{-\frac{\pi k M^{2}}{|e E|}}
$$

where $E$ is the electric field and $e, j, M$ are the charge, spin and mass of the pair created particles, and $d$ is the number of uncompact spacetime dimensions.

The phenomenon has been shown to hold in open superstring theory [2]. In this case, there is no gravity at tree level and the uniform electric field with flat spacetime geometry is a consistent background of the theory. In $d$ uncompact dimensions, one finds a result which at weak fields approaches the Schwinger formula (1.1) for the infinite collection of particles of the open superstring spectrum.

An important question is whether the phenomenon persists in the presence of gravity, where the back reaction to electric field configurations can play an important role. There are no static uniform electromagnetic field configurations in gravity. The basic reason is that electromagnetic flux lines attract each other and tend to cluster around some central region. Examples of axially symmetric, but non-uniform magnetic field configurations in 
closed (bosonic and super) string theory have been constructed and investigated in [3, 44. In particular, there is a two-parameter $(b, \tilde{b})$ class of models given by [3, 迆

$$
\begin{gathered}
d s^{2}=-d x_{0}^{2}+d r^{2}+\frac{r^{2}}{1+\tilde{b}^{2} r^{2}}(d \varphi+(b+\tilde{b}) d y)(d \varphi+(b-\tilde{b}) d y)+d y^{2}+d x_{1}^{2}+\ldots+d x_{D-4}^{2} \\
e^{-2\left(\phi-\phi_{0}\right)}=1+\tilde{b}^{2} r^{2}, \quad B_{2}=\tilde{b} \frac{r^{2}}{1+\tilde{b}^{2} r^{2}} d \varphi \wedge d y
\end{gathered}
$$

Here $y$ is a periodic coordinate, $y=y+2 \pi R, D=10$ for type II superstring and $D=26$ for closed bosonic string theory. More generally, this background solves the equations of the action

$$
S_{D}=\int d^{D} x \sqrt{G} e^{-2 \phi}\left(R+4\left(\partial_{\mu} \phi\right)^{2}-\frac{1}{12}\left(H_{\mu \nu \rho}\right)^{2}\right)
$$

in any spacetime dimensions.

Dimensional reduction in the $y$ direction gives two magnetic fields of different $U(1)$ 's, proportional to $b$ and $\tilde{b}$, associated with $g_{y \varphi}$ and $B_{y \varphi}$, respectively. T-duality exchanges the parameters $b$ and $\tilde{b}$. Setting the parameter $\tilde{b}$ to zero, one finds the Kaluza-Klein (KK) Melvin solution found in [5] and subsequently investigated in [6] in the context of gravitational field theories. The background (1.2) is an exact solution of (super)string theory to all $\alpha^{\prime}$ orders and the corresponding string conformal sigma model can be solved exactly [3, 4]. In particular, the full physical string spectrum can be obtained in terms of creation and annihilation operators, much like in the free string case.

As pointed out in [3], and investigated in [0, 8, 9, 10] for the model with $\tilde{b}=0$, electric field configurations can be obtained from (1.2) by a Wick rotation in the coordinates $x_{0} \rightarrow i x_{D-3}$ and $\varphi \rightarrow-i t$, so that $t$ now plays the role of time. By setting the parameter $\tilde{b}$ to zero and changing $b \rightarrow i E$ one finds the electric version of the Kaluza-Klein Melvin model, which we shall call the $E$-model

$$
E: d s^{2}=d r^{2}-r^{2}(d t-E d y)^{2}+d y^{2}+d x_{i} d x_{i}, \quad i=1, \ldots, D-3
$$

with constant dilaton and vanishing $B_{2}$-field. The space is locally flat, but it has non-trivial identifications.

The T-dual solution, that we shall call $\tilde{E}$-model, is obtained from (1.2) by setting $b=0$ and changing $\tilde{b} \rightarrow i \tilde{E}$,

$$
\begin{aligned}
\tilde{E}: \quad & d s^{2}=d r^{2}-\frac{r^{2}}{1-\tilde{E}^{2} r^{2}} d t^{2}+\frac{d y^{2}}{1-\tilde{E}^{2} r^{2}}+d x_{i} d x_{i} \\
e^{-2\left(\phi-\phi_{0}\right)} & =1-\tilde{E}^{2} r^{2}, \quad B_{2}=\tilde{E} \frac{r^{2}}{1-\tilde{E}^{2} r^{2}} d t \wedge d y,
\end{aligned}
$$

The geometry is singular at $r=1 / \tilde{E}$. Nonetheless, the string model is regular, as follows from the fact that T-duality gives an equivalent conformal field theory and the $E$-model (1.4) is obviously regular.

String states are characterized by their (integer) winding number $m$ and KK momentum $n$ in the compact direction $y$ as well as by quantum numbers representing internal 
excitations. The T-duality that maps the $E$-model to the $\tilde{E}$-model exchanges $n$ and $m$, so that a given string state of winding and KK momentum charges $(m, n)$ behaves in the $E$-model in exactly the same way as a string state with charges $(n, m)$ (changing, at the same time, $\left.R \rightarrow \alpha^{\prime} / R\right)$. In any of the two models, the presence of either charge, $m$ or $n$ produces a non-trivial interaction with the electric field.

The geometry (1.4) was investigated in the context of General Relativity in [10], where no Schwinger pair production for KK particles was found (though it was found some particle creation of different origin). The interpretation of this fact was that the back reaction of geometry prevents the electrostatic potential from overcoming the rest mass of the KK particles, preventing the tunneling that would otherwise give rise to the Schwinger effect. An interesting question is whether this is a model-dependent result or we should expect that gravitational back reaction will always prevent the standard Schwinger phenomenon to take place.

The present results clarify this point. In consistency with [10], we will show that in the $E$-model indeed there cannot be any Schwinger pair production of KK particles. However, we find that there is Schwinger pair production for winding string states. Such states cannot be investigated within the context of field theory, but one can consider the $\tilde{E}$-model, where these winding states become KK particles. Our results imply that in General Relativity coupled to a dilaton and an antisymmetric tensor $B_{2}$ as in (1.3) there is Schwinger pair production of KK particles in the background (1.5), as we shall discuss below. In this case we recall that the electric field arises from the $B_{t y}$ component.

This paper is organized as follows.

In section 2 we review the Schwinger effect for charged scalar particles, first in the more familiar case of an electric field in Minkowski spacetime [1] (see [11, 12, 13] for more recent discussions); then for an electric field in Rindler spacetime [14].

In section 3 we compute the pair creation rate for a charged scalar field in the $\tilde{E}$ model (1.5). We find that the probability for pair creation is given by

$$
\begin{gathered}
\mathcal{W}=\frac{\mathcal{T} V_{d-2}}{(2 \pi)^{d-1}|\tilde{E}|} \sum_{k=1}^{\infty} \frac{(-1)^{k+1}}{k}\left(\frac{2|q \tilde{E}|}{k}\right)^{\frac{d-1}{2}} e^{-\frac{\pi k M_{0}^{2}}{2|q \tilde{E}|}} \operatorname{erf}(Y \sqrt{k}) \\
Y \equiv \sqrt{\frac{\pi|q \tilde{E}|}{2 \tilde{E}^{2}}},
\end{gathered}
$$

where $\operatorname{erf}(z)$ is the standard error function and $q=n / R$ is the Kaluza-Klein charge of the state. $M_{0}$ is the mass in the higher $D$ dimensional spacetime and the mass in $d \equiv D-1$ dimensions is $M^{2}=M_{0}^{2}+q^{2}$. $\mathcal{W}$ exhibits a number of interesting physical features that we discuss.

In section 4 we discuss string theory in the background (1.4). In section 4.1, we first reproduce the formula of section 3 for (unexcited) winding string states. Then, in section 4.2 , we comment on some issues to derive a formula for general excited winding string states. 


\section{Charged scalar field}

The solution (1.5) represents an electric field on a space which approaches a Rindler space at small $r$. Before considering this space, it is useful to recall some features of the dynamics of charged particles coupled to an electric field in Minkowski and in Rindler spacetime.

\subsection{Electric field in Minkowski spacetime}

To study Schwinger pair creation in flat Minkowski spacetime, $d s^{2}=-d t^{2}+d z^{2}+d x_{i}^{2}$, $i=1, \ldots, d-2$, we choose a gauge $A_{t}=E z$ and consider the equation for a massive charged scalar particle minimally coupled to the electromagnetic field,

$$
\left(-\partial_{z}^{2}-\partial_{i}^{2}+\left(\partial_{t}-i e E z\right)^{2}+M^{2}\right) \Phi=0
$$

Setting $\Phi=e^{i p_{i} x_{i}+i \omega t} \chi(z)$ one gets the Schrödinger equation for a particle in an inverted harmonic potential $V=-(e E z-\omega)^{2}$,

$$
\left[-\partial_{z}^{2}-(e E z-\omega)^{2}+p_{i}^{2}+M^{2}\right] \chi(z)=0
$$

There are many different derivations of the Schwinger rate (1.1). In this first example we will compute the pair production rate from the partition function for the magnetic model obtained by analytic continuation, $t=i x, B=i E, \omega=-i p, x_{d-2}=-i x_{0}, p_{d-2}=i p_{0}$. This gives a harmonic oscillator with Hamiltonian

$$
H=-\partial_{z}^{2}+(e B z-p)^{2}+p_{j}^{2}-p_{0}^{2}+M^{2}, \quad j=1, \ldots, d-3
$$

and eigenvalues (we take $e B>0$ )

$$
H_{n}=2 e B\left(n+\frac{1}{2}\right)+p_{j}^{2}-p_{0}^{2}+M^{2}, \quad n=0,1,2, \ldots
$$

This leads to a one-loop vacuum energy

$$
Z=\int_{0}^{\infty} \frac{d s}{s} \operatorname{Tr}\left[e^{-\pi s H}\right]
$$

The trace contains a summation over the momenta, which is converted into an integral by the rule

$$
\sum_{p_{j}, p_{0}, p} \rightarrow \frac{V_{d-1}}{(2 \pi)^{d-1}} \int d^{d-3} p_{j} d p_{0} d p
$$

The integral over $d p$ gives a $\delta(0)$ which is interpreted as a time volume factor (recall that $-i p$ is the energy in the electric configuration). This is just what is needed to get a finite probability per unit time; since $d p$ has the same dimensions as $e B d t$, the integral over $d p$ gives a factor $e B T$. Thus

$$
Z=\frac{T V_{d-1}}{(2 \pi)^{d-1}} \int_{0}^{\infty} \frac{d s}{s^{\frac{d}{2}}} \frac{e B}{\sinh (\pi e B s)} e^{-\pi M^{2} s}
$$

In doing the analytic continuation back to the original electric field configuration, an infinite series of poles arise from $\sin (\pi e E s)=0$, i.e. at $s=k /(e E), k=1,2, \ldots$ which give rise 
to an imaginary part, given by $\pi$ times the residue of the poles (the integration contour passes by the right of the poles). This represents the pair production probability We get

$$
\mathcal{W}_{\text {scalar }}=2 \operatorname{Im} Z=\frac{T V_{d-1}}{(2 \pi)^{d-1}} \sum_{k=1}^{\infty}(-1)^{k+1}\left(\frac{|e E|}{k}\right)^{\frac{d}{2}} e^{-\frac{\pi k M^{2}}{|e E|}}
$$

in agreement with (1.1) with $j=0$. This is equivalent to the Lorentzian approach based on the Schwinger representation of the Feynman propagator by means of the kernel.

Another equivalent way to compute the pair production rate is to choose the gauge where the gauge potential is $A_{3}=-E x_{0}$. The equation of motion is then similar, changing $z$ by $x_{0}$, but now we can interpret pair production in terms of a scattering process, where an "in" wave hits the inverted harmonic potential and as a result there is a combination of negative and positive frequency waves at late times, where the coefficients are determined by a Bogoliubov transformation. The coefficient of the negative frequency component gives the pair creation probability for a given frequency. The similar calculation in the static gauge will be carried out in section 3 .

\subsection{Electric field in Rindler space}

The case of Rindler space is obtained as follows. We first consider a charged scalar particle moving in a uniform magnetic field in flat spacetime, but now we use radial coordinates and choose the gauge where $A_{\varphi}=B r^{2} / 2$ (i.e. $A_{x}=-B y / 2, A_{y}=B x / 2$ ). The equation is now given by

$$
\left(\frac{1}{r} \partial_{r} r \partial_{r}+\frac{1}{r^{2}}\left(\partial_{\varphi}-\frac{i}{2} e B r^{2}\right)^{2}+\partial_{j}^{2}-\partial_{0}^{2}-M^{2}\right) \Phi=0
$$

where $j=1, \ldots, d-3$. Setting $\Phi=e^{i p_{0} x_{0}+i l \varphi+i p_{j} x_{j}} \chi(r) / \sqrt{r}$, we obtain

$$
\left[-\partial_{r}^{2}+V(r)\right] \chi(r)=0, \quad V(r)=\left(\frac{1}{2} e B r-\frac{l}{r}\right)^{2}-\frac{1}{4 r^{2}}+M^{2}+p_{j}^{2}-p_{0}^{2}
$$

This represents a two-dimensional oscillator. The eigenvalues are

$$
\begin{aligned}
H & =e B\left(l_{L}+l_{R}+1\right)-e B\left(l_{L}-l_{R}\right)+M^{2}+p_{j}^{2}-p_{0}^{2}, \\
& =e B\left(2 l_{R}+1\right)+M^{2}+p_{j}^{2}-p_{0}^{2}, \quad l_{L}, l_{R}=0,1,2, \ldots
\end{aligned}
$$

where the quantum numbers are related to the Landau level $l$ and to the radial quantum number $k_{r}$ by

$$
l=l_{L}-l_{R}, \quad l_{L}+l_{R}=2 k_{r}+|l|, \quad k_{r}=0,1,2, \ldots
$$

One can then compute the partition function and analytically continue back to the electric field configuration, which is now obtained by analytic continuation $B=i E, \varphi=-i t, x_{0}=$ $i x_{d-2}$ and $l=i \omega$, giving the Rindler space

$$
d s^{2}=d r^{2}-r^{2} d t^{2}+d x_{i}^{2}, \quad i=1, \ldots, d-2 .
$$


Although the starting "uniform magnetic field" configuration is the same as in sect. 2.1, the analytic continuation is different: now it is the polar angle what becomes the time coordinate, giving as a result a Rindler space, with a Rindler horizon located at $r=0$. As shown in [14], in Rindler space one again obtains the Schwinger pair creation rate, modulo a term that scales as the area and represents Unruh particle production from the Rindler horizon. The rate can be obtained by a Bogoliubov transformation between creation/annihilation operators associated with in and out vacua at $r=0$ and $r=\infty$. Generalizing the result of [14 to $d$ dimensions, the formula for the rate (re-obtained in the next section) is given by

$$
\begin{aligned}
\mathcal{W} & =\frac{\mathcal{T} V_{d-2}}{(2 \pi)^{d-1}} \int d^{d-2} p \int_{-\infty}^{0} d \omega \log \frac{1+e^{-\frac{\pi\left(M^{2}+p_{i}^{2}\right)}{e E}}}{1+e^{-\frac{\pi\left(M^{2}+p_{i}^{2}\right)}{e E}} e^{2 \pi \omega}} \\
& =\mathcal{W}_{1}-\mathcal{W}_{2}
\end{aligned}
$$

where

$$
\begin{aligned}
& \mathcal{W}_{1}=\frac{\mathcal{T} V_{d-2}}{(2 \pi)^{d-1}} \int d^{d-2} p \int_{-\infty}^{0} d \omega \log \left(1+e^{-\frac{\pi\left(M^{2}+p_{i}^{2}\right)}{e E}}\right) \\
& \mathcal{W}_{2}=\frac{\mathcal{T} V_{d-2}}{(2 \pi)^{d-1}} \int d^{d-2} p \int_{-\infty}^{0} d \omega \log \left(1+e^{-\frac{\pi\left(M^{2}+p_{i}^{2}\right)}{e E}} e^{2 \pi \omega}\right)
\end{aligned}
$$

We assume $e E>0$. Expanding the log and performing the integrals, one finds

$$
\mathcal{W}_{1}=\frac{V_{R} V_{d-2}}{(2 \pi)^{d-1}} \sum_{k=1}^{\infty}(-1)^{k+1}\left(\frac{e E}{k}\right)^{\frac{d}{2}} e^{-\frac{\pi k M^{2}}{e E}}
$$

We used the relation 14 $\mathcal{T} d \omega=e E d V_{R}$, where $V_{R}$ is the volume in the two-dimensional Rindler spacetime. This can be regularized by two limiting hyperbolas at $r_{1}$ and $r_{2}$. Then one can write $V_{R}=T\left(r_{2}-r_{1}\right)$, where $T$ is a mean proper time, $T=\mathcal{T} \bar{r}, \bar{r}=\left(r_{1}+r_{2}\right) / 2$ representing a typical distance from the Rindler horizon (typical values are given by the turning points of classical trajectories in the WKB approximation). Thus $\mathcal{W}_{1}$ represents the standard Schwinger probability for pair creation proportional to the spacetime volume $V_{d-2} V_{R}$.

The second contribution is a surface contribution, which has to be subtracted to the dominant contribution (2.15) proportional to the volume. The integral over $\omega$ is now convergent, giving the result

$$
\mathcal{W}_{2}=\frac{\mathcal{T} V_{d-2}}{(2 \pi)^{d}} \int d^{d-2} p \sum_{k=1}^{\infty} \frac{(-1)^{k+1}}{k^{2}} e^{-\frac{\pi k\left(M^{2}+p_{i}^{2}\right)}{e E}}
$$

Integrating over $p_{i}$, we find

$$
\begin{aligned}
\mathcal{W}_{2} & =\frac{\mathcal{T} V_{d-2}}{(2 \pi)^{d}} \sum_{k=1}^{\infty} \frac{(-1)^{k+1}}{k^{2}}\left(\frac{e E}{k}\right)^{\frac{d}{2}-1} e^{-\frac{\pi k M^{2}}{e E}} \\
& =-\frac{\mathcal{T} V_{d-2}}{(2 \pi)^{d}}(e E)^{\frac{d}{2}-1} \operatorname{Li}_{1+\frac{d}{2}}\left(-e^{-\frac{\pi M^{2}}{e E}}\right)
\end{aligned}
$$


This second contribution is due to the presence of the Rindler horizon [14]. In the absence of electric fields, this term plays the role of canceling the particle production in the Boulware vacuum state. When the electric field is turned on, one term becomes proportional to the volume and the surface term becomes significant only in the vicinity of the horizon, where it is getting most of the contribution.

\section{Electric field in a gravitational theory}

Thus far we have considered electric fields in fixed (Minkowski or Rindler) backgrounds, and neglected the back reaction of the geometry due to the energy density provided by the electric field. We will now incorporate this back reaction exactly by using the string model (1.5).

We shall first consider a massless scalar supergravity mode $\Phi$ in the background (1.2) This satisfies the equation ${ }^{1}$

$$
\partial_{\mu}\left(e^{-2 \phi} \sqrt{G} G^{\mu \nu} \partial_{\nu}\right) \Phi=0
$$

Using eq. (1.2) we obtain

$$
\begin{aligned}
& {\left[-\partial_{0}^{2}+\partial_{k}^{2}+\frac{1}{r} \partial_{r}\left(r \partial_{r}\right)+\frac{1}{r^{2}}\left(1+b^{2} r^{2}\right)\left(1+\tilde{b}^{2} r^{2}\right) \partial_{\varphi}^{2}\right.} \\
& \left.+\left(1+\tilde{b}^{2} r^{2}\right) \partial_{y}^{2}-2 b\left(1+\tilde{b}^{2} r^{2}\right) \partial_{\varphi} \partial_{y}\right] \Phi=0
\end{aligned}
$$

with $k=1, \ldots, D-4$. Write

$$
\Phi=e^{i p_{0} x_{0}+i p_{k} x_{k}+i q y+i l \varphi} \frac{1}{\sqrt{r}} \eta(r), \quad q=\frac{n}{R}, \quad n \in \mathbb{Z}
$$

Then eq. (3.2) becomes

$$
\left[-\partial_{r}^{2}+V(r)\right] \eta(r)=0, \quad V(r)=\frac{l^{2}-\frac{1}{4}}{r^{2}}+\nu^{2} r^{2}+\mu^{2},
$$

where

$$
\nu=\tilde{b}(q-b l), \quad \mu^{2}=p_{k}^{2}-p_{0}^{2}+(q-b l)^{2}+\tilde{b}^{2} l^{2} .
$$

This is a two-dimensional oscillator with frequency $\nu$. We thus obtain essentially the same differential equation as in the Rindler case, (2.10), but with different parameters.

This mode $\Phi$ has (Kaluza-Klein) momentum charge but vanishing winding number (winding states cannot be described by local fields). Upon analytic continuation to the electric field configuration one finds an inverted harmonic oscillator potential $-|\nu|^{2} r^{2}$, which is again the origin of the instability that leads to Schwinger pair production. However, note that this term is absent in the $E$-model (1.4), where $\tilde{E}=0$, since $\nu=0$ in this case. This implies that no Schwinger pair production of Kaluza-Klein particles should be expected in this model. This explains the results of [10], which considered KK particles in the E-model (1.4).

\footnotetext{
${ }^{1}$ The same equation was considered in [15] in the study of the mass spectrum of the magnetic model.
} 
We now return to the magnetic variables and set $b=0$. Therefore we will examine pair production of Kaluza-Klein particles in the $\tilde{E}$-model. Then we get

$$
\nu=q \tilde{b}, \quad \mu^{2}=p_{k}^{2}-p_{0}^{2}+M^{2}+\tilde{b}^{2} l^{2}, \quad M=|q| .
$$

Since we started with the equation (3.1) for a massless scalar fluctuation, the invariant mass of the state in the lower dimensional theory in the absence of an electric field coincides with the electric charge of the field, $M=|q|$. Below we shall also consider massive states.

Now the Hamiltonian eigenvalues are

$$
\begin{gathered}
H=2 q \tilde{b}\left(l_{L}+l_{R}+1\right)+\tilde{b}^{2} l^{2}+M^{2}+p_{k}^{2}-p_{0}^{2}, \quad l_{L}, l_{R}=0,1,2, \ldots \\
M^{2} \equiv q^{2}=\frac{n^{2}}{R^{2}}, \quad l=l_{L}-l_{R}, \quad q \tilde{b}>0
\end{gathered}
$$

Comparing with the previous Minkowski and Rindler cases, the Hamiltonian now contains a new term $\tilde{b}^{2} l^{2}$. The origin of this term is the $\tilde{b}^{2} r^{2}$ term in the metric component $g^{\varphi \varphi}=$ $\left(1+\tilde{b}^{2} r^{2}\right) / r^{2}$. This term $\tilde{b}^{2} l^{2}$ is produced by the back reaction of the magnetic field in the geometry.

\subsection{Calculation of the pair-production rate}

Consider now the analytic continuation to the electric field configuration, $\tilde{b} \rightarrow i \tilde{E}$ and $l \rightarrow i \omega$, where $\omega$ is the Rindler energy. The calculation of vacuum persistence rate is formally the same as in [14], since the equation is the same with the substitutions

$$
e E \rightarrow 2 q \tilde{E}, \quad M^{2} \rightarrow M^{2}+\tilde{E}^{2} \omega^{2}-2 q \tilde{E} \omega
$$

It will be seen below that the new term $\tilde{E}^{2} \omega^{2}$ has a dramatic effect in the pair creation process. As mentioned above, this term originates from gravitational back reaction, whereas the second term is to cancel the term $-e B l \rightarrow 2 q \tilde{E} \omega$ in eq. (2.10), since this term does not appear in the differential equation (3.4). The absence of such term $2 q \tilde{E} \omega$ in (3.4) seems to be due to the Kaluza-Klein nature of the interaction.

The problem can be viewed as the standard quantum mechanical problem of scattering against a barrier (see e.g. [11, 12, 13]). The pair creation rate will be related to the reflection coefficient. The starting point is the differential equation satisfied by the wave function $\psi(r)=\eta(r) / \sqrt{r}$ :

$$
\psi^{\prime \prime}+\frac{1}{r} \psi^{\prime}+\left(q^{2} \tilde{E}^{2} r^{2}+\frac{\omega^{2}}{r^{2}}-\mu^{2}\right) \psi=0
$$

with

$$
\mu^{2}=M^{2}+p_{i}^{2}+\omega^{2} \tilde{E}^{2}, \quad M^{2}=q^{2}, \quad i=1, \ldots, D-3
$$

(whereas $\mu^{2}=M^{2}+p_{i}^{2}+e E \omega$ for the previous Rindler case). It is important to note that, despite the classical singularity of the background at $r=1 / \tilde{E}$, the wave equation is regular at this point and can be extrapolated beyond this radius.

It is convenient to introduce new variables (throughout we assume $q E>0$ )

$$
z=q \tilde{E} r^{2}, \quad \psi=z^{\frac{i}{2} \omega} e^{-i \frac{z}{2}} g(z)
$$


Then $g(z)$ satisfies the differential equation

$$
z g^{\prime \prime}(z)+(1+i \omega-i z) g^{\prime}(z)-\alpha g(z)=0
$$

with

$$
\alpha \equiv \frac{\mu^{2}}{4 q \tilde{E}}-\frac{\omega}{2}+\frac{i}{2}
$$

The solution is given in terms of the confluent hypergeometric function

$$
g(z)=A z^{-i \omega}{ }_{1} F_{1}(-i \alpha-i \omega, 1-i \omega ; i z)+B{ }_{1} F_{1}(-i \alpha, 1+i \omega ; i z) .
$$

Near $z=0, \psi(z)$ behaves as

$$
\psi(z) \cong A z^{-\frac{i}{2} \omega}+B z^{\frac{i}{2} \omega}, \quad z \sim 0 .
$$

Defining $z=e^{2 x}$, one can write the $z=0$ behavior in terms of incoming and outgoing ordinary plane waves,

$$
e^{i \omega t} \psi(x) \cong A e^{i \omega(t-x)}+B e^{i \omega(t+x)}, \quad x \rightarrow-\infty .
$$

At $z \rightarrow \infty$, $\psi$ has the following behavior

$$
\psi(z) \cong C z^{i \alpha+\frac{i}{2} \omega} e^{-i \frac{z}{2}}+D z^{-i \alpha-1-\frac{i}{2} \omega} e^{i \frac{z}{2}}, \quad z \gg 1
$$

where

$$
\begin{aligned}
& C=e^{\frac{\pi \alpha}{2}}\left(\frac{B \Gamma(1+i \omega)}{\Gamma(1+i \omega+i \alpha)}+\frac{A e^{\frac{\pi \omega}{2}} \Gamma(1-i \omega)}{\Gamma(1+i \alpha)}\right) \\
& D=e^{\frac{\pi(\alpha-i)}{2}}\left(\frac{B e^{\frac{\pi \omega}{2}} \Gamma(1+i \omega)}{\Gamma(-i \alpha)}+\frac{A \Gamma(1-i \omega)}{\Gamma(-i \alpha-i \omega)}\right)
\end{aligned}
$$

Therefore

$$
e^{i \omega t} \psi(z) \cong \frac{1}{\sqrt{z}} e^{i \omega t}\left(C z^{\frac{i \mu^{2}}{4 q E}} e^{-\frac{i z}{2}}+D z^{-\frac{i \mu^{2}}{4 q E}} e^{\frac{i z}{2}}\right)
$$

We must demand that there is no wave coming from infinity. For positive frequency modes, this is the condition $D \equiv 0$. This gives

$$
\left|\frac{B}{A}\right|^{2}=\left|\frac{e^{-\frac{\pi \omega}{2}} \Gamma(-i \alpha)}{\Gamma(-i \alpha-i \omega)}\right|^{2}=\frac{1+e^{-\frac{\pi\left(M^{2}+p_{i}^{2}\right)}{2 q \tilde{E}}} e^{-\pi\left(\frac{\omega^{2} \tilde{E}^{2}}{2 q \tilde{E}}+\omega\right)}}{1+e^{-\frac{\pi\left(M^{2}+p_{i}^{2}\right)}{2 q \tilde{E}}} e^{-\pi\left(\frac{\omega^{2} \tilde{E}^{2}}{2 q \tilde{E}}-\omega\right)}}<1
$$

For negative frequency, the condition is $C \equiv 0$, and we get an equivalent result:

$$
\left|\frac{B}{A}\right|^{2}=\left|\frac{e^{\frac{\pi \omega}{2}} \Gamma(1+i \omega+i \alpha)}{\Gamma(1+i \alpha)}\right|^{2}=\frac{1+e^{-\frac{\pi\left(M^{2}+p_{i}^{2}\right)}{2 q \tilde{E}}} e^{-\pi\left(\frac{\omega^{2} \tilde{E}^{2}}{2 q \tilde{E}}-\omega\right)}}{1+e^{-\frac{\pi\left(M^{2}+p_{i}^{2}\right)}{2 q \tilde{E}}} e^{-\pi\left(\frac{\omega^{2} \tilde{E}^{2}}{2 q \tilde{E}}+\omega\right)}}<1
$$

The Rindler space, as well as the present spacetime (1.5), can be divided in four quadrants, left, right, future and past. As shown in [14], the sum $2 \sum_{\omega>0}+2 \sum_{\omega<0}$ gives the total 
volume. Quantization in the right quadrant corresponds to keeping only $\omega<0$. If we were to integrate over both regions, $\omega>0$ and $\omega<0$, we would get an extra factor of 2 .

Equation (3.19) gives the reflection probability of a given mode, which is equivalent to the probability for vacuum persistence or vacuum to vacuum transition,

$$
\mid\langle 0, \text { out }| 0, \text { in }\rangle\left.\right|^{2}=\left|\frac{B}{A}\right|^{2}=\exp \left(-2 V T \operatorname{Im} \mathcal{L}_{\text {eff }}\right)
$$

where $\mathcal{L}_{\text {eff }}$ is the effective Lagrangian. The pair-production probability is then ${ }^{2}$

$$
\mathcal{W}=2 V T \operatorname{Im} \mathcal{L}_{\text {eff }}=-\log \left|\frac{B}{A}\right|^{2}
$$

Integrating over the momenta and over the frequency, the full pair creation probability is given by

$$
\mathcal{W}=\frac{\mathcal{T} V_{d-2}}{(2 \pi)^{d-1}} \int d^{d-2} p \int_{-\infty}^{0} d \omega \log \frac{1+e^{-\frac{\pi\left(M^{2}+p_{i}^{2}\right)}{2 q \tilde{E}}} e^{-\pi\left(\frac{\omega^{2} \tilde{E}^{2}}{2 q \tilde{E}}+\omega\right)}}{1+e^{-\frac{\pi\left(M^{2}+p_{i}^{2}\right)}{2 q E}} e^{-\pi\left(\frac{\omega^{2} \tilde{E}^{2}}{2 q E}-\omega\right)}}
$$

where $d=D-1$ is the number of uncompact spacetime dimensions. This exactly reproduces the result (2.14) of the Rindler model upon the formal substitution (3.6), as expected.

For the present, gravitational case, the physical picture now involves important differences, having to do with the presence of the factor $e^{-\frac{\pi \omega^{2} \tilde{E}^{2}}{2 q \tilde{E}}}$, induced by a gravitational correction $\Delta M^{2}=\omega^{2} E^{2}$ to the mass squared $M^{2}=q^{2}=n^{2} / R^{2}$ of the Kaluza-Klein particle. Expanding the logarithm and integrating over $p_{i}$, we now find

$$
\mathcal{W}=\mathcal{W}_{1}-\mathcal{W}_{2}
$$

with

$$
\begin{aligned}
& \mathcal{W}_{1}=\frac{\mathcal{T} V_{d-2}}{(2 \pi)^{d-1}} \sum_{k=1}^{\infty} \frac{(-1)^{k+1}}{2 q \tilde{E}}\left(\frac{2 q \tilde{E}}{k}\right)^{\frac{d}{2}} e^{-\frac{\pi k M^{2}}{2 q \tilde{E}}} \int_{-\infty}^{0} d \omega e^{-\pi k\left(\frac{\omega^{2} \tilde{E}^{2}}{2 q \tilde{E}}+\omega\right)} \\
& \mathcal{W}_{2}=\frac{\mathcal{T} V_{d-2}}{(2 \pi)^{d-1}} \sum_{k=1}^{\infty} \frac{(-1)^{k+1}}{2 q \tilde{E}}\left(\frac{2 q \tilde{E}}{k}\right)^{\frac{d}{2}} e^{-\frac{\pi k M^{2}}{2 q \tilde{E}}} \int_{-\infty}^{0} d \omega e^{-\pi k\left(\frac{\omega^{2} \tilde{\tilde{E}}^{2}}{2 q \tilde{E}}-\omega\right)}
\end{aligned}
$$

Integrating over $\omega$, we obtain

$$
\begin{aligned}
& \mathcal{W}_{1}=\frac{\mathcal{T} V_{d-2}}{2(2 \pi)^{d-1} \tilde{E}} \sum_{k=1}^{\infty} \frac{(-1)^{k+1}}{k}\left(\frac{2 q \tilde{E}}{k}\right)^{\frac{d-1}{2}} e^{-\frac{\pi k\left(M^{2}-q^{2}\right)}{2 q \tilde{E}}}(1+\operatorname{erf}(Y \sqrt{k})) \\
& \mathcal{W}_{2}=\frac{\mathcal{T} V_{d-2}}{2(2 \pi)^{d-1} \tilde{E}} \sum_{k=1}^{\infty} \frac{(-1)^{k+1}}{k}\left(\frac{2 q \tilde{E}}{k}\right)^{\frac{d-1}{2}} e^{-\frac{\pi k\left(M^{2}-q^{2}\right)}{2 q \tilde{E}}}(1-\operatorname{erf}(Y \sqrt{k}))
\end{aligned}
$$

\footnotetext{
${ }^{2}$ Note that $\mathcal{W} \equiv 2 V T \operatorname{Im} \mathcal{L}_{\text {eff }}$ has the interpretation of pair-production probability only to leading order in the semiclassical approximation, while eq.(3.20) is an exact relation (for a discussion see [16]). We thank S. Gavrilov for emphasizing this point.
} 


$$
Y \equiv \sqrt{\frac{\pi q \tilde{E}}{2 \tilde{E}^{2}}}
$$

where $\operatorname{erf}(z)$ is the standard error function. Remarkably, the integrals over $\omega$ are convergent: there is no infinite volume factor coming from the radial coordinate. Both $\mathcal{W}_{1}$ and $\mathcal{W}_{2}$ are proportional to $\mathcal{T} V_{d-2}$, just like the surface term $\mathcal{W}_{2}$ in the Rindler case of section 2.2. Near the horizon $\mathcal{T} \sim T / \bar{r}$, where as in the Rindler case $T$ is proper time and $\bar{r}$ is a characteristic distance, so the pair production rate $\mathcal{W} / T$ goes roughly like $1 / \bar{r}$, becoming more important in the vicinity of the horizon.

Since in the present case $M^{2}=q^{2}$, there is an exact cancellation of two exponential factors appearing in $\mathcal{W}_{1}$ and $\mathcal{W}_{2}$. Therefore

$$
\begin{aligned}
& \mathcal{W}_{1}=\frac{\mathcal{T} V_{d-2}}{2(2 \pi)^{d-1} \tilde{E}} \sum_{k=1}^{\infty} \frac{(-1)^{k+1}}{k}\left(\frac{2 q \tilde{E}}{k}\right)^{\frac{d-1}{2}}(1+\operatorname{erf}(Y \sqrt{k})), \\
& \mathcal{W}_{2}=\frac{\mathcal{T} V_{d-2}}{2(2 \pi)^{d-1} \tilde{E}} \sum_{k=1}^{\infty} \frac{(-1)^{k+1}}{k}\left(\frac{2 q \tilde{E}}{k}\right)^{\frac{d-1}{2}}(1-\operatorname{erf}(Y \sqrt{k})) .
\end{aligned}
$$

Now consider particles with $M^{2}>q^{2}$. Our starting point is a scalar field with action

$$
S=\int d^{D} x \sqrt{G} e^{-2 \phi}\left(G^{\mu \nu} \partial_{\mu} \Phi \partial_{\nu} \Phi+M_{0}^{2} \Phi^{2}\right) .
$$

The equation of motion is given by

$$
\partial_{\mu}\left(e^{-2 \phi} \sqrt{G} G^{\mu \nu} \partial_{\nu}\right) \Phi=\sqrt{G} e^{-2 \phi} M_{0}^{2} \Phi
$$

For the background (1.2), the differential equation is the same as (3.2) with a term $M_{0}^{2} \Phi$ on the right hand side. The subsequent equations are the same with the substitution of $M^{2}$ by $M^{2} \equiv M_{0}^{2}+q^{2}$. Thus, proceeding in the same way as above, the general pair creation rate is then

$$
\mathcal{W}=\frac{\mathcal{T} V_{d-2}}{(2 \pi)^{d-1} \tilde{E}} \sum_{k=1}^{\infty} \frac{(-1)^{k+1}}{k}\left(\frac{2 q \tilde{E}}{k}\right)^{\frac{d-1}{2}} e^{-\frac{\pi k M_{0}^{2}}{2 q \tilde{E}}} \operatorname{erf}(Y \sqrt{k})
$$

This is the main result of this paper.

\subsection{General properties}

The sums over $k$ are convergent for both $\mathcal{W}_{1}$ and $\mathcal{W}_{2}$. This is seen from the behavior of the error function $\operatorname{erf}(z)$ : it is monotonically increasing with a linear behavior near $z \sim 0$ and tending to 1 at $z \gg 1$. The asymptotic expansion of the error function is given by

$$
\sqrt{\pi} e^{z^{2}}(1-\operatorname{erf}(z))=\sum_{n=0}^{\infty} \frac{(-1)^{n}}{2^{n} z^{2 n+1}}(2 n-1) ! !
$$

Therefore, for any fixed electric field and $k \gg 1$, we can substitute $\operatorname{erf}(Y \sqrt{k}) \rightarrow 1$ and the series (3.30) is readily seen to be convergent for the cases of interest, viz. $d \geq 2$ (including the case $M_{0}=0$, eqs. (3.26), (3.27)). 
For weak electric fields, $Y$ is large, and we find

$$
\begin{aligned}
& (1+\operatorname{erf}(Y \sqrt{k})) \cong 2+O\left(e^{-k Y^{2}}\right) \\
& (1-\operatorname{erf}(Y \sqrt{k})) \cong \frac{1}{\sqrt{\pi k} Y} e^{-\frac{\pi k q \tilde{E}}{2 \tilde{E}^{2}}}
\end{aligned}
$$

In this limit $\mathcal{W}_{2}$ becomes negligible. The dominant contribution to the rate is

$$
\mathcal{W} \cong \mathcal{W}_{1} \cong \frac{\mathcal{T} V_{d-2}}{(2 \pi)^{d-1} \tilde{E}} \sum_{k=1}^{\infty} \frac{(-1)^{k+1}}{k}\left(\frac{2 q \tilde{E}}{k}\right)^{\frac{d-1}{2}} e^{-\frac{\pi k\left(M^{2}-q^{2}\right)}{2 q \tilde{E}}}
$$

Thus, at weak fields, the probability for pair creation for particles with $M>|q|$ has the same structure as in the Schwinger formula (1.1). More precisely, one has a relation of the form $\mathcal{W}_{\text {grav }}^{(d)}\left(M_{0}^{2}\right)=$ const. $\frac{1}{2 q \tilde{E}^{2}} \mathcal{W}_{\text {schw }}^{(d+1)}\left(M_{0}^{2}\right)$.

Interestingly, for the case $M=|q|$, the rate is not exponentially suppressed. We obtain

$$
\begin{aligned}
\mathcal{W} & \cong \frac{\mathcal{T} V_{d-2}}{(2 \pi)^{d-1} \tilde{E}} \sum_{k=1}^{\infty} \frac{(-1)^{k+1}}{k}\left(\frac{2 q \tilde{E}}{k}\right)^{\frac{d-1}{2}} \\
& \cong \frac{\mathcal{T} V_{d-2}}{(2 \pi)^{d-1} \tilde{E}} c_{0}(2 q \tilde{E})^{\frac{d-1}{2}}, \quad c_{0}=\left(1-2^{\frac{1-d}{2}}\right) \zeta\left(\frac{d}{2}+\frac{1}{2}\right)
\end{aligned}
$$

This seems to be related to the fact that such state is massless in the original higher dimensional geometry.

Now let us examine the behavior of the pair creation rate at strong electric fields. In this limit, $Y$ is small. The argument of the error function is small for $k \ll k_{0} \sim O(E)$. Since the series is convergent, terms with $k>k_{0}$ will give a subleading contribution. For the terms with $k \ll k_{0}$ that provide the leading contribution, one has

$$
\operatorname{erf}(Y \sqrt{k})=\frac{2}{\sqrt{\pi}} Y \sqrt{k}+O\left(Y^{3}\right) \cong \sqrt{\frac{2 k q \tilde{E}}{\tilde{E}^{2}}}
$$

Therefore we find

$$
\begin{aligned}
\mathcal{W} & \cong \frac{\mathcal{T} V_{d-2}}{(2 \pi)^{d-1} \tilde{E}} \sum_{k=1}^{\infty} \frac{(-1)^{k+1}}{k}\left(\frac{2 q \tilde{E}}{k}\right)^{\frac{d-1}{2}} \sqrt{\frac{2 k q \tilde{E}}{\tilde{E}^{2}}} \\
& \cong \frac{\mathcal{T} V_{d-2}}{(2 \pi)^{d-1} \tilde{E}^{2}} c_{0}^{\prime}(2 q \tilde{E})^{\frac{d}{2}}, \quad c_{0}^{\prime}=\left(1-2^{1-\frac{d}{2}}\right) \zeta\left(\frac{d}{2}\right), \quad d \neq 2,
\end{aligned}
$$

and $c_{0}^{\prime}=\log (2)$ for $d=2$. Note that this is the leading behavior also for particles with $M>|q|$. In the particularly interesting case of $d=4$ the rate goes to a constant in the strong field limit:

$$
\left.\mathcal{W}_{d=4}\right|_{\tilde{E}=\infty}=\mathcal{T} V_{2} \frac{q^{2}}{24 \pi} .
$$

Comparing the weak and strong field behaviors of $M=|q|$ particles, for weak fields we have $\mathcal{W} \sim E^{\frac{d-3}{2}}$ whereas for strong fields $\mathcal{W} \sim E^{\frac{d-4}{2}}$. Therefore, for these particles, the strong field behavior in $d$ dimensions is the same as the weak field behavior in $d^{\prime}=d-1$. 
Summarizing, the behavior of $\mathcal{W}$ for particles with $M=|q|$ is as follows.

- For $d=2 \mathcal{W}$ is divergent as $\tilde{E} \rightarrow 0$ (see (3.34)) and goes to zero like $1 / \tilde{E}$ as $\tilde{E} \rightarrow \infty$. The divergence at zero field may look puzzling, since for weak fields, gravitational back reaction is small and one would expect to recover results similar to those of the model of sect. 2.2, which is regular as $E \rightarrow 0$, even for particles with $M=0$. This will be clarified in the next section.

- For $d=3, \mathcal{W}$ has a finite, non-vanishing value at $\tilde{E}=0$, given by $\mathcal{W}=\mathcal{T} V_{1} q / 24$. As $\tilde{E}$ is increased, the rate decreases monotonically and, as $\tilde{E} \rightarrow \infty$, it goes to zero like $1 / \sqrt{\tilde{E}}$.

- For $d=4, \mathcal{W}$ is monotonically increasing, beginning from 0 at $\tilde{E}=0$ and approaching a constant $\mathcal{T} V_{2} q^{2} /(24 \pi)$ as $\tilde{E} \rightarrow \infty$.

- For $d>4, \mathcal{W}$ is monotonically increasing, beginning from 0 at $\tilde{E}=0$ and going to infinity as $\tilde{E} \rightarrow \infty$.

For particles with $M>|q|$, the leading behavior at $\tilde{E}=\infty$ is the same as that of particles with $M=|q|$. The weak field behavior, given in eq. (3.33), is quite different, instead. As $\tilde{E} \rightarrow 0, \mathcal{W}$ vanishes exponentially as $\exp \left(-\pi M_{0}^{2} /(2 q E)\right)$ in all dimensions. In particular, this shows that for $d=2,3$, the rate has a maximum at some finite value of the electric field (since for these values of $d, \mathcal{W}$ vanishes also at $\tilde{E}=\infty$ ).

\subsection{Discussion}

To connect with the Rindler case of sect. 2.2 , we have to consider a situation where the gravitational correction $\Delta M^{2}=\omega^{2} \tilde{E}^{2}$ can be neglected. In particular, it should be much smaller than the mass squared $M^{2}=M_{0}^{2}+q^{2}$, which is the case for modes with frequency $\omega^{2} \ll M^{2} / \tilde{E}^{2}$. Therefore we shall come back to eq. (3.22) and integrate over $\omega$ with a cutoff at some $\omega_{0}^{2} \ll M^{2} / \tilde{E}^{2}$. Expanding the log and integrating over $p_{i}$, we have (see eq. (3.24)

$$
\mathcal{W}=\frac{\mathcal{T} V_{d-2}}{(2 \pi)^{d-1}} \sum_{k=1}^{\infty} \frac{(-1)^{k+1}}{2 q E}\left(\frac{2 q \tilde{E}}{k}\right)^{\frac{d}{2}} \int_{-\left|\omega_{0}\right|}^{0} d \omega e^{-\frac{\pi k M^{2}}{2 q \tilde{E}}} e^{-\frac{\pi k \omega^{2} \tilde{E}^{2}}{2 q \tilde{E}}}\left(e^{-\pi k \omega}-e^{\pi k \omega}\right)
$$

Define $\epsilon \equiv \omega_{0} \tilde{E} / M$. Then for $\omega \sim \omega_{0}$ the exponent in $(3.38)$ is ${ }^{3}$

$$
-\frac{\pi k M^{2}}{2 q \tilde{E}}-\frac{\pi k \omega^{2} \tilde{E}^{2}}{2 q \tilde{E}} \pm \pi k \omega \sim-\frac{\pi k M^{2}}{2 q \tilde{E}}\left(1+\epsilon^{2} \pm \frac{2 q}{M} \epsilon\right)
$$

The gravitational back reaction term, proportional to $\epsilon^{2}$, can be neglected provided $\epsilon \ll 1$ and $\epsilon \ll q / M$, the latter being in general a stronger condition since $M \geq q$. This requires $\left|\omega_{0}\right| \ll q / \tilde{E}$. In this limit we have

$$
\begin{aligned}
\mathcal{W} & \cong \frac{\mathcal{T} V_{d-2}}{(2 \pi)^{d-1}} \sum_{k=1}^{\infty} \frac{(-1)^{k+1}}{2 q E}\left(\frac{2 q \tilde{E}}{k}\right)^{\frac{d}{2}} \int_{-\left|\omega_{0}\right|}^{0} d \omega e^{-\frac{\pi k M^{2}}{2 q \tilde{E}}}\left(e^{-\pi k \omega}-e^{\pi k \omega}\right) \\
& \cong \frac{4 \mathcal{T} V_{d-2}}{(2 \pi)^{d}} \sum_{k=1}^{\infty} \frac{(-1)^{k+1}}{k^{2}}\left(\frac{2 q \tilde{E}}{k}\right)^{\frac{d-2}{2}} e^{-\frac{\pi k M^{2}}{2 q \tilde{E}}}\left(\cosh \left(\pi k \omega_{0}\right)-1\right)
\end{aligned}
$$

\footnotetext{
${ }^{3}$ Here we assume both $q>0$ and $\tilde{E}>0$. The cases of $q<0$ and/or $\tilde{E}<0$ are obtained by replacing $q$ and $\tilde{E}$ by $|q|$ and $|\tilde{E}|$ in $\mathcal{W}$.
} 
Note that for these modes with $|\omega|<\left|\omega_{0}\right|$ it is $M^{2}$, rather than $M^{2}-q^{2}$, what appears in the exponent.

In the Rindler case (2.13), restricting the integrations to the same modes with $|\omega|<$ $\left|\omega_{0}\right| \ll M^{2} / e E$, one obtains

$$
\mathcal{W}=\frac{\mathcal{T} V_{d-2}}{(2 \pi)^{d-1}} \sum_{k=1}^{\infty} \frac{(-1)^{k+1}}{2 q E}\left(\frac{2 q \tilde{E}}{k}\right)^{\frac{d}{2}} \int_{-\left|\omega_{0}\right|}^{0} d \omega e^{-\frac{\pi k M^{2}}{2 q \tilde{E}}}\left(1-e^{2 \pi k \omega}\right)
$$

In general, this is different from (3.40), as expected, since the models are different. Nevertheless, it is interesting that it gives the same result as (3.40) when the integral is restricted to low energy modes, $\omega_{0} \ll 1$, i.e. the probabilities agree precisely in the regime where the rate is significant. To see this, we note that, because of the exponential factor, $\mathcal{W}$ in $(3.40)$ is very small unless $\frac{M^{2}}{q \tilde{E}}<O(1)$. Since $\omega \ll \frac{q}{\tilde{E}} \leq \frac{M^{2}}{q \tilde{E}}$, this condition implies $\omega_{0} \ll 1$, i.e. low-frequency modes. In this case we can assume $k \omega_{0} \ll 1$ and expand the $\cosh \left(\pi k \omega_{0}\right){ }^{4}$ We find

$$
\mathcal{W}=\frac{\pi \mathcal{T} V_{d-2}}{(2 \pi)^{d-1}} \sum_{k=1}^{\infty}(-1)^{k+1}\left(\frac{2 q \tilde{E}}{k}\right)^{\frac{d-2}{2}} e^{-\frac{\pi k M^{2}}{2 q \tilde{E}}} \omega_{0}^{2},
$$

which exactly agrees with (3.41) in the same limit.

The formula (3.40) also clarifies the apparent puzzle mentioned above concerning the singular behavior of eq. (3.34) for $d=2$ at zero $\tilde{E}$. This divergence originates from modes with large $\omega$, for which the gravitational back reaction term $\omega^{2} \tilde{E}^{2}$ is important. In restricting the integration to modes for which the gravitational back reaction is small, we find (3.40) which has a regular zero field limit in any dimensions, as expected.

In the present case it is not clear how to disentangle the Unruh effect from the Schwinger process, since there is no infinite radial volume factor. The Unruh effect should be significant near the horizon, whereas typically the Schwinger effect appears in the region where the electric field has a non-vanishing value, giving a rate typically proportional to the volume of this region. We recall that the geometry (1.5) of the present model is nontrivial. The pair creation process arises as the net result of this complicated combination of Rindler horizon with gauge fields and back reaction. It is possible that it makes no sense to attempt to separate Unruh and Schwinger effects at any value of the electric field.

\section{Bosonic string model}

The partition function for the two-parameter $(b, \tilde{b})$ magnetic model (1.2) in bosonic string theory was found in [3]. The models with $(b, 0)$ and $(0, \tilde{b})$, being related by T-duality, have identical partition function with the exchange of $R \rightarrow \alpha^{\prime} / R$. Here we will set $\tilde{b}=0$ and use units where $\alpha^{\prime}=1$. One gets

$$
Z=c_{1} \int_{\mathcal{F}} \frac{d^{2} \tau}{\tau_{2}^{13}} e^{4 \pi \tau_{2}}\left|f\left(e^{2 i \pi \tau}\right)\right|^{-48} \sum_{\left(w^{\prime}, m\right) \neq(0,0)} \exp \left(-\frac{\pi R^{2}}{\tau_{2}}\left|w^{\prime}-\tau m\right|^{2}\right)
$$

\footnotetext{
${ }^{4}$ Since the series is convergent, large values of $k$ with $k>O\left(1 / \omega_{0}\right)$ give subleading contributions.
} 


$$
\times e^{-\frac{\pi(\chi-\bar{\chi})^{2}}{2 \tau_{2}}} \frac{1}{|\sin (\pi \chi)|^{2}} \prod_{r=1}^{\infty} \frac{\left|\left(1-e^{2 \pi i r \tau}\right)\right|^{4}}{\left|\left(1-e^{2 \pi i(r \tau+\chi)}\right)\left(1-e^{2 \pi i(r \tau-\chi)}\right)\right|^{2}}
$$

where

$$
\begin{gathered}
\chi=b R\left(w^{\prime}-\tau m\right), \quad \bar{\chi}=b R\left(w^{\prime}-\bar{\tau} m\right), \quad b>0 . \\
f\left(e^{2 i \pi \tau}\right)=\prod_{r=1}^{\infty}\left(1-e^{2 i r \pi \tau}\right), \quad c_{1}=\frac{V_{D-3} R}{4(2 \pi)^{D-3}}, \quad D=26 .
\end{gathered}
$$

Here $\mathcal{F}$ denotes as usual the fundamental domain of $S L(2, Z)$ defined by $\mathcal{F}=\left\{\left|\tau_{1}\right| \leq\right.$ $\left.1 / 2,|\tau|^{2} \geq 1\right\}$ in the upper half-plane $\tau_{2}>0$. The partition function (4.1) has a divergent zero field limit corresponding to the area of the plane $\left(x_{1}, x_{2}\right) \equiv(r, \varphi)$. Alternatively, one could project out the factor coming from the integral of the constant modes of $x_{1}, x_{2}$ (this prescription was adopted in [3]). This leads to (4.1) with an additional factor $|\chi|^{2} / \tau_{2}$. In both cases the partition function is modular invariant.

Before discussing some features of the general structure of this partition function, it is useful to study it in the particle limit and compare with the results of the previous section. In the T-dual language that we are studying, with $\tilde{b}=0$ and $b \neq 0$, this Kaluza-Klein particle corresponds to a winding state.

\subsection{Schwinger effect for winding string states}

In order to obtain the partition function for the winding state from (4.1), the first step is to drop the factors $\left|f\left(e^{2 i \pi \tau}\right)\right|$ and the factors in the product from $r=1$ to $\infty$ associated with string excitations. Next, by a Poisson resummation in $w^{\prime}$, i.e. by using the formula

$$
\sum_{w^{\prime}} F\left(w^{\prime}\right)=\sum_{n} \int_{-\infty}^{\infty} d \mu e^{2 \pi i n \mu} F(\mu)
$$

we go to the Hamiltonian representation where the physical states are exhibited explicitly. We are interested in states with $m \neq 0$ and $n=0$. Also, in the field theory limit, the integral over the fundamental domain region is to be extended to the full strip $\left|\tau_{1}\right| \leq \frac{1}{2}$, $\tau_{2}>0$. By a change of integration variable, $x=\mu-m \tau_{1}$, the integration over $\tau_{1}$ is trivial (equal to 1$)$ and we finally obtain $\left(s \equiv \tau_{2}\right)$

$$
Z_{\text {particle }}=2 c_{1} \int_{0}^{\infty} \frac{d s}{s^{13}} \int_{-\infty}^{\infty} d x e^{-\frac{\pi R^{2} x^{2}}{s}} \sum_{m=1}^{\infty} e^{-\pi s\left(R^{2} m^{2}-2 b^{2} R^{2} m^{2}-4\right)} \frac{1}{|\sin (\pi b R(x-i m s))|^{2}}
$$

The terms $-2 b^{2} R^{2} m^{2}-4$ in the exponent come from normal ordering of the Hamiltonian, including all string oscillators [3]. Therefore they will be dropped in what follows, since they do not arise in the particle theory (these terms are absent in the supersymmetric theory, see [4] and below).

To make contact with the spectrum (3.5), we have to expand the sine function. We get

$Z_{\text {particle }}=8 c_{1} \int_{0}^{\infty} \frac{d s}{s^{13}} \int_{-\infty}^{\infty} d x e^{-\frac{\pi R^{2} x^{2}}{s}} \sum_{m=1}^{\infty} e^{-\pi s R^{2} m^{2}} \sum_{l_{L}, l_{R}=0}^{\infty} e^{-2 \pi s m b R\left(l_{L}+l_{R}+1\right)} e^{2 \pi i x b R\left(l_{L}-l_{R}\right)}$ 
Integrating over $x$, we find

$$
Z_{\text {particle }}=\frac{8 c_{1}}{R} \sum_{m=1}^{\infty} \int_{0}^{\infty} \frac{d s}{s^{25 / 2}} \sum_{l_{L}, l_{R}=0}^{\infty} e^{-\pi s\left(R^{2} m^{2}+b^{2}\left(l_{L}-l_{R}\right)^{2}\right)} e^{-2 \pi s m b R\left(l_{L}+l_{R}+1\right)}
$$

This is what one would find from a Hamiltonian

$$
H=2 m b R\left(l_{L}+l_{R}+1\right)+R^{2} m^{2}+b^{2}\left(l_{L}-l_{R}\right)^{2}+p_{\mu}^{2}, \quad \mu=0, \ldots, D-4, \quad D=26 .
$$

Changing $b \rightarrow \tilde{b}, R \rightarrow 1 / R$ and $m \rightarrow n$, this is exactly the Hamiltonian (3.5).

Recalling

$$
l=l_{L}-l_{R}, \quad l_{L}+l_{R}=2 k_{r}+|l|, \quad k_{r}=0,1,2, \ldots,
$$

we write

$$
\sum_{l_{L}, l_{R}=0}^{\infty}=\sum_{l=0}^{\infty} \sum_{k_{r}=0}^{\infty}+\sum_{l=-\infty}^{-1} \sum_{k_{r}=1}^{\infty}
$$

Then we can write $Z_{\text {particle }}=Z_{+}+Z_{-}$with

$$
\begin{aligned}
& Z_{+}=\frac{8 c_{1}}{R} \sum_{m=1}^{\infty} \sum_{l=0}^{\infty} \sum_{k_{r}=0}^{\infty} \int_{0}^{\infty} \frac{d s}{s^{25 / 2}} e^{-\pi s\left(R^{2} m^{2}+b^{2} l^{2}\right)} e^{-2 \pi s m b R\left(2 k_{r}+l+1\right)} \\
& Z_{-}=\frac{8 c_{1}}{R} \sum_{m=1}^{\infty} \sum_{l=-\infty}^{-1} \sum_{k_{r}=1}^{\infty} \int_{0}^{\infty} \frac{d s}{s^{25 / 2}} e^{-\pi s\left(R^{2} m^{2}+b^{2} l^{2}\right)} e^{-2 \pi s m b R\left(2 k_{r}-l+1\right)}
\end{aligned}
$$

Summing over $k_{r}$, we find

$$
\begin{aligned}
& Z_{+}=\frac{4 c_{1}}{R} \sum_{m=1}^{\infty} \sum_{l=0}^{\infty} \int_{0}^{\infty} \frac{d s}{s^{25 / 2}} e^{-\pi s\left(R^{2} m^{2}+b^{2} l^{2}\right)} e^{-2 \pi s m b R l} \frac{1}{\sinh (2 \pi s m b R)} \\
& Z_{-}=\frac{4 c_{1}}{R} \sum_{m=1}^{\infty} \sum_{l=-\infty}^{-1} \int_{0}^{\infty} \frac{d s}{s^{25 / 2}} e^{-\pi s\left(R^{2} m^{2}+b^{2} l^{2}\right)} e^{2 \pi s m b R l} \frac{1}{\sinh (2 \pi s m b R)}
\end{aligned}
$$

Now consider the analytic continuation to the Lorentzian electric field configuration. This requires an additional prescription which is not supplied by the Euclidean magnetic partition function. In section 3 we have seen that, in going to the Lorentzian electric field configuration $b \rightarrow i E$, at the same time we have to change $l \rightarrow i \omega$, and $\omega$ is a continuous variable representing the Rindler energy of a given mode. One has to be careful in the Wick rotation $l \rightarrow i \omega$, so we will proceed in two steps. We first replace $l$ by a continuous variable $\ell$. We have

$$
\begin{aligned}
& Z_{+}=\frac{\mathcal{T}}{2 \pi} \frac{4 c_{1}}{R} \sum_{m=1}^{\infty} \int_{0}^{\infty} d \ell \int_{0}^{\infty} \frac{d s}{s^{25 / 2}} e^{-\pi s\left(R^{2} m^{2}+b^{2} \ell^{2}\right)} e^{-2 \pi s m b R \ell} \frac{1}{\sinh (2 \pi s m b R)} \\
& Z_{-}=\frac{\mathcal{T}}{2 \pi} \frac{4 c_{1}}{R} \sum_{m=1}^{\infty} \int_{-\infty}^{0} d \ell \int_{0}^{\infty} \frac{d s}{s^{25 / 2}} e^{-\pi s\left(R^{2} m^{2}+b^{2} \ell^{2}\right)} e^{2 \pi s m b R \ell} \frac{1}{\sinh (2 \pi s m b R)}
\end{aligned}
$$


We have used the same normalization as before for the density of states. The Wick rotation $\ell \rightarrow i \omega$, for both regions of integrations $\ell>0$ and $\ell<0$ must be done to the negative imaginary axes for $\omega$, since, as discussed in the quantum field theory treatment, the negative frequency modes are those contributing to the rate in the quantization in the right quadrant. Starting with clockwise oriented contours, there will be a relative sign between $Z_{+}$and $Z_{-}$ contributions because in one case one has to reverse the sense of integration of $\omega$. The result is

$$
\begin{aligned}
& Z_{+}=\frac{2 \mathcal{T} c_{1}}{\pi R} \sum_{m=1}^{\infty} \int_{-\infty}^{0} d \omega \int_{0}^{\infty} \frac{d s}{s^{25 / 2}} e^{-\pi s\left(R^{2} m^{2}+E^{2} \omega^{2}\right)} e^{2 \pi s m E R \omega} \frac{1}{\sin (2 \pi s m E R)} \\
& Z_{-}=-\frac{2 \mathcal{T} c_{1}}{\pi R} \sum_{m=1}^{\infty} \int_{-\infty}^{0} d \omega \int_{0}^{\infty} \frac{d s}{s^{25 / 2}} e^{-\pi s\left(R^{2} m^{2}+E^{2} \omega^{2}\right)} e^{-2 \pi s m E R \omega} \frac{1}{\sin (2 \pi s m E R)}(4 .
\end{aligned}
$$

The pair creation rate is equal to $2 \operatorname{Im}\left(Z_{\text {particle }}\right)$. The imaginary part arises from the poles of $1 / \sin (2 \pi s m E R)$. As dictated by the definition of the Feynman propagator, the integration contour passes by the right of the poles, and the imaginary part is then given by $\pi$ times the residue at the poles,

$$
s_{0}=\frac{k}{2 m E R}, \quad \operatorname{Res}_{s_{0}} \frac{1}{\sin (2 \pi s m E R)}=\frac{(-1)^{k}}{2 \pi m E R} .
$$

Thus we find

$$
\begin{aligned}
& Z_{+}=\frac{\mathcal{T} V_{d-2}}{(2 \pi)^{d-1}} \sum_{m=1}^{\infty} \sum_{k=1}^{\infty} \int_{-\infty}^{0} d \omega \frac{(-1)^{k}}{2 m E R}\left(\frac{2 m E R}{k}\right)^{\frac{25}{2}} e^{-\frac{\pi k}{2 m E R}\left(M^{2}+\omega^{2} E^{2}\right)} e^{\pi k \omega} \\
& Z_{-}=-\frac{\mathcal{T} V_{d-2}}{(2 \pi)^{d-1}} \sum_{m=1}^{\infty} \sum_{k=1}^{\infty} \int_{-\infty}^{0} d \omega \frac{(-1)^{k}}{2 m E R}\left(\frac{2 m E R}{k}\right)^{\frac{25}{2}} e^{-\frac{\pi k}{2 m E R}\left(M^{2}+\omega^{2} E^{2}\right)} e^{-\pi k \omega}
\end{aligned}
$$

with $M=m R$ and $d=D-1=25$. This exactly reproduces the result (3.24) found directly from the differential equation in the previous section (recall the T-dual dictionary: $E \rightarrow \tilde{E}, m \rightarrow n$ and $R \rightarrow 1 / R)$. The integral over $\omega$ can now be performed with identical results.

\subsection{Comments on the extension to general string states}

We now comment on the computation of the pair production rate for excited winding string states. In general, one expects that they should be pair produced since they are electrically charged as long as they have non-vanishing winding number. In sect. 4.1 we have seen that obtaining the electric model from the magnetic model by analytic continuation involves a number of subtleties. In particular, the orbital angular momentum $l$ becomes a continuous variable representing the energy of the modes. Finding the correct prescription for the complete partition function involves many new issues. Here we outline some aspects of the calculation. We start with (4.1) and perform Poisson resummation as in (4.4) to put the magnetic model in a suitable form for analytic continuation. Expand each of the factors in (4.1),

$$
\frac{1}{1-e^{2 \pi i(r \tau \pm \chi)}}=\sum_{N_{r}^{ \pm}=0}^{\infty} e^{2 \pi i N_{r}^{ \pm}(r \tau \pm \chi)}, \quad \chi=b R\left(x-i \tau_{2} m\right)
$$


with $x=\mu-m \tau_{1}$. We obtain

$$
\begin{aligned}
& Z=c_{1} \sum_{\text {states }} d_{\text {state }} \int_{\mathcal{F}} \frac{d^{2} \tau}{\tau_{2}^{13}} e^{2 \pi i\left(N_{R}-N_{L}-m w\right) \tau_{1}} \int_{-\infty}^{\infty} d x e^{-\frac{\pi R^{2} x^{2}}{\tau_{2}}+2 \pi i n x} \\
& \times e^{-\pi \tau_{2}\left(2 N_{R}+2 N_{L}+m^{2} R^{2}-2 m b R\left(J_{R}-J_{L}\right)-2 b^{2} R^{2} m^{2}-4\right)} e^{2 \pi i x b R\left(J_{R}+J_{L}\right)}
\end{aligned}
$$

where $d_{\text {state }}$ represents the degeneracy and

$$
N_{R, L}=\sum_{r=1}^{\infty} r\left(N_{r}^{+}+N_{r}^{-}\right)_{R, L}, \quad J_{R, L}=\mp\left(l_{R, L}+\frac{1}{2}\right)+\sum_{r=1}^{\infty}\left(N_{r}^{+}-N_{r}^{-}\right)_{R, L}
$$

Next, integrate over $x$ and get

$$
Z=\frac{c_{1}}{R} \sum_{\text {states }} d_{\text {state }} \int_{\mathcal{F}} \frac{d^{2} \tau}{\tau_{2}^{25 / 2}} e^{2 \pi i\left(N_{R}-N_{L}-m w\right) \tau_{1}} e^{-\pi \tau_{2} M^{2}}
$$

where

$$
M^{2}=2 N_{R}+2 N_{L}+m^{2} R^{2}+\left(\frac{n}{R}+b\left(J_{R}+J_{L}\right)\right)^{2}-2 m b R\left(J_{R}-J_{L}\right)-2 b^{2} R^{2} m^{2}-4
$$

This of course reproduces the mass spectrum found in [3]. The last two terms $-2 b^{2} R^{2} m^{2}-4$ imply the presence of the usual bosonic string tachyon. To avoid this problem, one can start with the superstring partition function [4]

$$
\begin{aligned}
& Z_{\text {susy }}=c \int_{\mathcal{F}} \frac{d^{2} \tau}{\tau_{2}^{5}} \sum_{\left(w^{\prime}, w\right) \neq(0,0)} \exp \left(-\frac{\pi R^{2}}{\tau_{2}}\left|w^{\prime}-\tau w\right|^{2}\right)\left|f\left(e^{2 i \pi \tau}\right)\right|^{-12} \\
& \times \frac{|\sin (\pi \chi / 2)|^{8}}{|\sin (\pi \chi)|^{2}} \prod_{n=1}^{\infty} \frac{\left|\left(1-e^{2 \pi i\left(n \tau+\frac{\chi}{2}\right)}\right)\left(1-e^{2 \pi i\left(n \tau-\frac{\chi}{2}\right)}\right)\right|^{8}}{\left|\left(1-e^{2 \pi i(n \tau+\chi)}\right)\left(1-e^{2 \pi i(n \tau-\chi)}\right)\right|^{2}}
\end{aligned}
$$

with $\chi$ defined in (4.2). Proceeding in the same way, one finds a similar formula as (4.18), with a different degeneracy $d_{\text {state }}^{\text {susy }}$, and with

$$
M_{\text {susy }}^{2}=2 N_{R}+2 N_{L}+m^{2} R^{2}+\left(\frac{n}{R}+b\left(\hat{J}_{R}+\hat{J}_{L}\right)\right)^{2}-2 m b R\left(\hat{J}_{R}-\hat{J}_{L}\right)
$$

Now the spectrum is tachyon free below some critical value of the magnetic field [4, 15] and the angular momentum operators $\hat{J}_{R}, \hat{J}_{L}$ take both integer and half-integer values (while $\left.N_{R}, N_{L}=0,1,2, \ldots\right)$.

The problem is how to implement analytic continuation to the electric field model in Lorentzian space. This seems to require $b \rightarrow i E$ and $J_{R}+J_{L} \rightarrow i\left(J_{R}+J_{L}\right)$, since the total angular momentum is the variable conjugate to $\varphi$ and $\varphi \rightarrow-i t$. From sect. 4.1, we know that this is the case for the zero mode part where $l \rightarrow i \omega$. It should be the case that the spectra of $\hat{J}_{R}+\hat{J}_{L}$ and $\hat{J}_{R}-\hat{J}_{L}$ operators contain continuous and discrete parts related to Rindler energies and radial modes.

One could attempt to directly quantize strings in the Lorentzian electric field configuration. This also involves a number of subtle issues (see [7, 8, 9] for discussions). In the magnetic model, the zero modes satisfy the algebra of creation and annihilation operators. 
There is an obvious definition of Fock space and a physical string spectrum which is easily understood: the magnetic field only introduces corrections to the mass of each string state of given quantum numbers [4]. In the electric field configuration, the zero modes satisfy a Heisenberg algebra and it is not clear how the Fock space should be defined in order to reproduce the rates found in the previous sections.

Alternatively, one may attempt to directly compute the Euclidean path integral using the conformal $\sigma$-model associated with the background (1.4), i.e. the Euclidean version of the $E$-model (1.4). This leads to the partition function (4.1) or (4.20) with the change $b \rightarrow i E$. This partition function with $b \rightarrow i E$, and without any additional change, does not appear to be the proper starting point to obtain the pair production rate. Such partition function (which appeared in the literature in the context of cosmological Milne universes in [7, 8, 9]), presents a number of features which are not well understood. It has an infinite number of poles in the interior of the fundamental domain that lead to logarithmic divergences. These poles can be more conveniently visualized on the strip $\left|\tau_{1}\right| \leq \frac{1}{2}, \tau_{2}>0$ by unfolding the fundamental domain: the pair $\left(w^{\prime}, m\right)$ can be written as $\left(w^{\prime}, m\right)=k(p, q)$ where $k$ is an integer and $p, q$ are relatively primes. Then, by a modular transformation, one can set $p$ or $q$ to zero and the sum over $(p, q)$ can be traded by a sum over copies of the fundamental domain, finally obtaining a total integration region given by the strip $\left|\tau_{1}\right| \leq \frac{1}{2}, \tau_{2}>0$. A similar situation arose for the first time in a different context [17]: strings propagating in thermal $A d S_{3}$ backgrounds (or $H_{3} / Z$ ), representing the Euclidean BTZ black hole. Indeed, the partition function of [17] is very similar to the bosonic string partition function (4.1). In that case, the divergences were interpreted as an infinite volume factor due to the fact that the long strings of $A d S_{3}$ feel a flat potential and can move to any radial position. For our present problem of strings in the space (1.4), it is not clear how the partition function (4.1) with $b \rightarrow i E$ could capture the Lorentzian physics, since in the Euclidean approach the time variable is treated as an angle and the conjugate quantum numbers (such as $l_{L, R}, S_{L, R}$ ) are discrete, while, as discussed above, a continuous part is expected. Clearly, it would be interesting to obtain a closed formula for the pair creation rate for the complete string theory spectrum.

\section{Acknowledgments}

We acknowledge support by MCYT FPA 2007-66665.

\section{References}

[1] J. S. Schwinger, "On gauge invariance and vacuum polarization," Phys. Rev. 82, 664 (1951).

[2] C. Bachas and M. Porrati, "Pair creation of open strings in an electric field," Phys. Lett. B 296, 77 (1992) [arXiv:hep-th/9209032].

[3] J. G. Russo and A. A. Tseytlin, "Exactly solvable string models of curved space-time backgrounds," Nucl. Phys. B 449, 91 (1995) [arXiv:hep-th/9502038].

[4] J. G. Russo and A. A. Tseytlin, "Magnetic flux tube models in superstring theory," Nucl. Phys. B 461, 131 (1996) [arXiv:hep-th/9508068]. 
[5] G. W. Gibbons and K. i. Maeda, "Black Holes And Membranes In Higher Dimensional Theories With Dilaton Fields," Nucl. Phys. B 298, 741 (1988).

[6] F. Dowker, J. P. Gauntlett, G. W. Gibbons and G. T. Horowitz, "The Decay of magnetic fields in Kaluza-Klein theory," Phys. Rev. D 52, 6929 (1995) [arXiv:hep-th/9507143].

[7] L. Cornalba and M. S. Costa, "A new cosmological scenario in string theory," Phys. Rev. D 66, 066001 (2002) [arXiv:hep-th/0203031].

[8] B. Pioline and M. Berkooz, "Strings in an electric field, and the Milne universe," JCAP 0311, 007 (2003) [arXiv:hep-th/0307280].

[9] M. Berkooz, B. Pioline and M. Rozali, "Closed strings in Misner space," JCAP 0408, 004 (2004) [arXiv:hep-th/0405126].

[10] T. Friedmann and H. L. Verlinde, "Schwinger pair creation of Kaluza-Klein particles: Pair creation without tunneling," Phys. Rev. D 71, 064018 (2005) [arXiv:hep-th/0212163].

[11] T. Padmanabhan, "Quantum Theory In External Electromagnetic And Gravitational Fields: A Comparison Of Some Conceptual Issues," Pramana 37, 179 (1991).

[12] K. Srinivasan and T. Padmanabhan, "A novel approach to particle production in an uniform electric field," arXiv:gr-qc/9911022.

[13] S. P. Kim and D. N. Page, "Schwinger pair production via instantons in a strong electric field," Phys. Rev. D 65, 105002 (2002) [arXiv:hep-th/0005078].

[14] C. Gabriel and P. Spindel, "Quantum charged fields in Rindler space," Annals Phys. 284, 263 (2000) [arXiv:gr-qc/9912016].

[15] J. G. Russo and A. A. Tseytlin, "Magnetic backgrounds and tachyonic instabilities in closed superstring theory and M-theory," Nucl. Phys. B 611, 93 (2001) [arXiv:hep-th/0104238].

[16] S. P. Gavrilov, D. M. Gitman and J. L. Tomazelli, "Density matrix of a quantum field in a particle-creating background," Nucl. Phys. B 795, 645 (2008) [arXiv:hep-th/0612064].

[17] J. M. Maldacena, H. Ooguri and J. Son, "Strings in AdS(3) and the SL(2,R) WZW model. II: Euclidean black hole," J. Math. Phys. 42, 2961 (2001) [arXiv:hep-th/0005183]. 